\title{
Antiinflammatorisch wirksame Phytotherapeutika und ihr mögliches Potential bei tumorkranken Menschen
}

\author{
Reinhard Saller $^{\mathrm{a}}$ Jörg Melzer ${ }^{\mathrm{a}}$ Matthias Rostock ${ }^{\mathrm{a}, \mathrm{b}}$ \\ ${ }^{a}$ Institut für Naturheilkunde, Universitätsspital, Zürich, Schweiz \\ ${ }^{\mathrm{b}}$ Universitäres Cancer Center Hamburg, Hubertus Wald Tumorzentrum, Universitätsklinikum Hamburg-Eppendorf, Deutschland
}

\section{Schlüsselwörter}

Phytotherapie - Vielstoffgemische - Tumorerkrankungen . Metastasierung · Antiinflammatorische Wirkungen ·

Pleiotropie $\cdot$ Multi-Target

\section{Zusammenfassung}

Die Wirkstoffe pflanzlicher Arznei- und Heilmittel sind pleiotrope Vielstoffgemische mit Multi-Target Eigenschaften einschließlich antiinflammatorischer Wirkungen. Eine pleiotrope Entzündungshemmung könnte bei tumorkranken Menschen als Versuch der Verhinderung bzw. Verzögerung der Metastasierung eine bedeutsame Rolle spielen. Zahlreiche experimentelle Daten für europäische wie auch außereuropäische Pflanzen und verschiedene phytotherapeutische Kombinationen weisen auf eine solche Möglichkeit hin. Trotz der bislang nur geringen Anzahl klinischer Untersuchungen könnten derartige phytotherapeutische Behandlungsversuche vertretbar erscheinen, wenn für solche Arznei- und Heilpflanzen aus Anwendungsbereichen bei nicht tumorkranken Menschen gesicherte Daten über Qualität und Sicherheit dokumentiert sind und eine Übertragung solcher Daten auf die Situation tumorkranker Menschen unter kritischer Abwägung möglich erscheint. Phytotherapeutika spielen zudem in der Supportivtherapie eine zunehmende Rolle, wobei zahlreiche dieser Phytotherapeutika neben ihrer symptomatischen supportiven Wirksamkeit auch antiinflammatorische Wirkungen zeigen. Die gezielte Auswahl von supportiv wirksamen Phytotherapeutika mit zusätzlichen antiinflammatorischen Effekten könnte für tumorkranke Menschen neben der Symptomlinderung simultan die Möglichkeit einer antiinflammatorischen antitumoralen Wirksamkeit bieten als eine Art personalisierter Phytotherapie. Zurzeit besteht diesbezüglich noch ein großer Bedarf an therapeutisch orientierter klinischer Forschung.

\section{Keywords}

Phytotherapy · Multi-ingredient compounds - Cancer . Metastasis - Antiinflammatory effects .

Pleiotropic effects - Multi-target

\section{Summary \\ Antiinflammatory Herbal Drugs and Their Therapeutic Potential in Tumor Patients}

The active components of herbal drugs and substances are pleiotropic multi-ingredient compounds with multitarget properties including antiinflammatory effects. A pleiotropic inhibition of inflammation could play an important role in tumor patients as an attempt of prevention or retardation of metastasis. A large number of experimental data for European and non-European herbal drugs as well as various herbal drug combinations suggest such a possibility. Despite the so far small number of clinical studies, such an experimental herbal treatment could appear to be reasonable and acceptable, provided that there are data available on quality and safety of these herbal drugs by treatments of patients without cancer and provided that an application of these data seems to be justified. Besides, herbal drugs and substances play a growing role in supportive therapy. Many of these herbal drugs have antiinflammatory effects beside their symptomatic efficacy in supportive therapy. The specific selection of herbal drugs that are efficacious in supportive therapy and additionally showed antiinflammatory effects offers the possibility of simultaneous antiinflammatory and antitumor efficacy beside the symptomatic relief for tumor patients as personalized herbal treatment. However, at present there is still a great need and demand for therapy-oriented clinical research.

\section{KARGER \\ Fax +497614520714 \\ Information@Karger.de}

www.karger.com

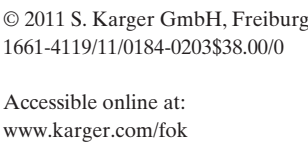




\section{Einleitung}

Heilmittel aus Pflanzen gehören zu den menschheitsgeschichtlich ältesten Therapieformen. Zahlreiche der bereits in der Vergangenheit verwendeten Heilpflanzen werden auch gegenwärtig zur Herstellung pflanzlicher Arznei- und Heilmittel im Rahmen der verschiedenen Richtungen der modernen Medizin verwendet [1-3]. Der Gebrauch pflanzlicher Heilmittel lässt sich medizin- und pharmaziegeschichtlich in praktisch allen Medizinsystemen trotz unterschiedlicher kultureller und geographischer Prägung nachweisen [4-6]. Eine erhebliche Anzahl der Heilpflanzen und die davon therapeutisch verwendeten Pflanzenteile (Drogen; zumeist getrocknete Pflanzenteile) werden mit nachvollziehbarer Kontinuität seit mehreren Jahrhunderten, wenn nicht einigen Jahrtausenden, angewendet $[4,5,7-10]$. Teilweise sind die symptombezogenen Anwendungsgebiete der Drogen trotz erheblicher Änderungen von Medizinkonzepten und -theorien relativ konstant geblieben $[2,3,5,7,8,10,11]$. Zu den Medizinsystemen mit einer langen, dokumentierten, phytotherapeutischen Tradition und gleichzeitiger moderner Bedeutung des pflanzlichen Arzneischatzes gehören unter anderem die Traditionelle Chinesische Medizin (TCM), die Ayurvedische Medizin, aber auch die Traditionelle Europäische Naturheilkunde bzw. Medizin (TEN, TEM; Tab. 1).

Eine Reihe von Heilpflanzen wurde nur in einem der Medizinsysteme verwendet, nicht wenige jedoch in mehreren Therapiesystemen. In der Gegenwart werden viele von ihnen global verwendet $[3,11,12]$. Zudem fand über lange Zeiträume und teils große Entfernungen ein derzeit vielfach vergessener intensiver Austausch, vor allem von Pflanzen und Drogen, zwischen diesen Medizinsystemen statt.

\section{Pflanzliche Wirkstoffe}

Heilmittel aus Pflanzen und pflanzliche Arzneimittel (Phytotherapeutika) unterscheiden sich in wesentlichen Gesichtspunkten von anderen modernen derzeit zumeist chemischsynthetischen Arzneimitteln, aber auch von sogenannten Biologicals. Sie sind genuine (phytogene) Vielstoffgemische und nicht Einzelsubstanzen oder einfache Kombinationen von Monosubstanzen [1,13-16]. Dies führt zu relevanten wissenschaftlichen, aber auch praktisch-therapeutischen Konsequenzen $[15,17]$. Der Wirkstoff (d.h. das Vielstoffgemisch) muss z.B. über die Inhaltsstoffanalyse hinaus auch funktionell betrachtet werden, die reine Analyse der Einzelkomponenten ergäbe kein hinreichendes Funktionsbild. Die Wirkstoffkomponenten gehen untereinander vielfältige strukturelle und funktionelle, aber auch plastische, zumeist schwache Bindungen ein. Die Gesamtheit der zahlreichen funktionell-plastischen Interaktionsmöglichkeiten der Einzelkomponenten untereinander können im Sinne eines flexiblen Netzwerks auf die zahlreichen Targets des Organismus einwirken (netzwerkartige phytotherapeutische Wirkmechanismen) [17]. $\mathrm{Zu}$ den Haupt-Targets gehören Proteine, DNA, RNA sowie dazugehörige Enzyme, Transskriptionsfaktoren und Biomembranen $[14,15,18]$.

Der Wirkungsmechanismus eines solchen Wirkstoffs weist auf eine genuine Pleiotropie hin [14, 15, 17]. Pleiotropie heißt, dass sich der gesamthafte Wirkungsmechanismus aus einer Reihe voneinander weitgehend unabhängiger Wirkmechanismen zusammensetzt bzw. dass bei einem pleiotropen Wirkstoff mehrere von einander unabhängige Wirkmechanismen vorliegen. Ein solcher komplexer Wirkstoff kann multifokal bzw. als Arzneimittel mit Multi-Target-Eigenschaften wirken. Dementsprechend erweisen sich solche Wirkstoffe in der Regel als nichtselektiv. Eine solche Nichtselektivität, d.h. ein relativer Multi-Target-Ansatz mit breiter
Tab. 1. Weltweit verwendete Arzneiund Heilpflanzen

\begin{tabular}{ll}
\hline Vorkommen & Umgang mit Arznei- und Heilpflanzen \\
\hline Europa & Über 350 Arznei- und Heilpflanzen als Rohstoffe für Phytotherapeutika mit zum Teil mehreren \\
Weltweit & Unterarten und zum Teil zahlreichen Sorten (nach derzeit weitgehend europäischen Traditionen) \\
& Gesicherte bzw. potentielle Arznei- und Heilpflanzen: über 35000 Arten \\
& Arznei- und Heilpflanzen außereuropäischer Medizinkulturen (z.B. Ayurvedische Medizin, TCM - \\
& 500 Pflanzen im Arzneibuch, zirka 7000 Pflanzen in der volksmedizinischen Anwendung, d.h. zirka \\
& 20\% der chinesischen Flora). Teilweise Übernahme indischer, chinesischer und tibetischer Arznei- \\
& drogen nach Europa (Überschneidungen zwischen chinesischem, tibetischem und ayurvedischem \\
& Heilpflanzenschatz) \\
& Verwendung von Monodrogen und vor allem vielfältiger Kombinationen pflanzlicher Drogen \\
& Verwendung von Phytotherapeutika (Qualität und Status nach dem jeweiligen Arzneimittelrecht) \\
& bzw. von Supplementen (Botanicals, Neutraceuticals; Qualität und Status bestenfalls nach dem \\
& jeweiligen Lebensmittelrecht) \\
& Aufnahme Heilpflanzen mit der Nahrung: teilweise Überlappung mit gezielt ausgewählten vege- \\
& tarischen Anteilen der Ernährung (insgesamt $>5000$ Pflanzenarten, davon 2300 kultiviert, davon \\
& zirka 150 ökonomisch bedeutsam, zirka 20 als Hauptanteil) \\
\hline TCM = Traditionelle & Chinesische Medizin. \\
\hline
\end{tabular}

Forsch Komplementmed 2011;18:203-212
Saller/Melzer/Rostock 
Tab. 2. Charakteristika des phytotherapeutischen Wirkstoffs

\begin{tabular}{ll}
\hline Bezeichnung & Charakteristika \\
\hline Genuines Vielstoffgemisch & $\begin{array}{l}\text { Sowohl in Einzeldrogen bzw. Einzelextrakten als auch in } \\
\text { Drogenkombinationen und Extraktkombinationen }\end{array}$ \\
\hline Strukturelles Vielstoffgemisch & $\begin{array}{l}\text { Komplex multipler Bestandteile (Wirkstoffkomponenten in } \\
\text { meist sehr niedrigen Konzentrationen) }\end{array}$ \\
\hline Funktionelles Vielstoffgemisch & $\begin{array}{l}\text { Wirkungsgefüge aufgrund vielfältiger Effekte und drogen- } \\
\text { bzw. organismusbezogener Interaktionsmöglichkeiten } \\
\text { (Netzwerkmodell, Plastizität) }\end{array}$ \\
& $\begin{array}{l}\text { Wirkstoff mit multiplen und multimodalen Wirkungen } \\
\text { (multifokal, Multi-Target) } \\
\text { Pleiotrope Eigenschaften (mehrere bis viele voneinander } \\
\text { unabhängige Wirkmechanismen) } \\
\text { Indikationsbezogene Anwendung (Indikation als Sonderfall } \\
\text { bzw. Teilaspekt des phytotherapeutischen Potentials) } \\
\text { Anwendung als potentielles «Systemmittel» (z.B. Ausnutzung } \\
\text { antiinflammatorischer Eigenschaften bei ätiopathogenetisch } \\
\text { bedeutsamen chronischen Entzündungsvorgängen) }\end{array}$ \\
\hline
\end{tabular}

biologischer Aktivität, könnte durchaus erhebliche Vorteile bieten, nicht zuletzt, da viele Krankheiten und auch Tumorerkrankungen auf dem komplexen Zusammenspiel zahlreicher, zum Teil noch nicht genauer charakterisierter Targets beruhen. Multi-Target-Eigenschaften und Pleiotropie könnten zudem beim häufigen Vorliegen von Komorbiditäten und bei Multimorbiditäten [17] im Rahmen einer Tumorerkrankung bedeutsam sein. Dabei ließen sich als potenziell attraktiver Behandlungsansatz durchaus nichtselektive Multi-Target-Wirkstoffe mit selektiven Mono-Target-Wirkstoffen kombinieren. Eine gewisse Nichtselektivität und Pleiotropie liegt, nicht zuletzt aus evolutionären Gründen, auch bereits bei zahlreichen sekundären Pflanzenstoffen (Monosubstanzen) vor, die die Bestandteile der phytotherapeutischen Wirkstoffe darstellen [14, 15].

Die Einzelkomponenten des Wirkstoffs liegen zumeist in sehr geringen Konzentrationen vor, so dass in der Regel nicht das gesamte dosisabhängige quantitative Potential dieser Komponenten zum Tragen kommt (z.B. keine vollständige Stimulation, Hemmung, Up- oder Down-Regulation). Eine Reihe von Einzelkomponenten pflanzlicher Wirkstoffe sind praktisch ubiquitär in Pflanzen, bzw. kommen in zahlreichen unterschiedlichen Arznei- und Heilpflanzen vor. Daher ist es nicht ungewöhnlich, dass verschiedene pflanzliche Wirkstoffe gemeinsame Teilwirkungen aufweisen. So gibt es zahlreiche pflanzliche Wirkstoffe in Arznei- und Heilmitteln, die einerseits gemeinsame antientzündliche Eigenschaften besitzen (eine Art Systemeigenschaft), andererseits sich aber in weiteren Wirkungen voneinander unterscheiden.

Aufgrund des Vielstoffcharakters haben pflanzliche Arznei- und Heilmittel in qualitativer Hinsicht ein zumeist breiteres Wirkungsspektum als Monosubstanzen, häufig auch ein breiteres Anwendungsspektrum (therapeutischer MultiTarget-Ansatz). So lässt sich z.B. auch, je nach therapeu- tischen Erfordernissen und je nach weiterer behandlungsbedürftiger Symptomatik relativ patientenzentriert, aus der Vielzahl von pflanzlichen Mitteln mit antientzündlichen Eigenschaften ein Mittel auswählen, das mehrere der bei einem Patienten gegebenen Behandlungsanlässe umfassen kann, z.B. Johanniskrautextrakte mit antientzündlichen und antidepressiven Wirkungen [13, 19]. Diese Vorgehensweise lässt sich als eine Art personalisierte patientenzentrierte Phytotherapie charakterisieren. Tabelle 2 fasst wesentliche Eigenheiten eines phytotherapeutischen Wirkstoffs zusammen.

\section{Phytotherapeutische Behandlungen bei tumorkranken Menschen}

Tumorkranke Menschen beziehen zu einem großen Prozentsatz $(20-80 \%)$ komplementärmedizinische bzw. naturheilkundliche Behandlungsansätze aus unterschiedlichen, zumeist komplexen Gründen in die Behandlung ihrer Tumorerkrankung bzw. der krankheitsbedingten Beeinträchtigungen und Einschränkungen mit ein. Die Einbeziehung von Komplementärmedizin bedeutet in der Regel keinen Verzicht auf moderne onkologische Behandlungsansätze. Einen Schwerpunkt komplementärmedizinischer zusätzlicher Behandlungen stellen Arznei- und Heilmittel aus Pflanzen dar. Sehr häufig stehen supportive Gesichtspunkte im Vordergrund [20, 21], z.B. die Behandlung von Müdigkeit und Erschöpfung sowie gastrointestinaler Symptome [22], die Vorbeugung und Behandlung von unerwünschten Wirkungen moderner onkologischer Behandlungen, «Entgiftungsansätze» im Nachgang von z.B. Zytostase, Radiotherapie und Behandlungen auf dem weiten Feld vielfältiger Maßnahmen, die körper- und personenspezifische «Abwehr» zu stärken [23]. Gründe für die gezielte Verwendung von Zubereitungen aus Heilpflanzen 
im Verlauf einer Tumorerkrankung, die Patienten, aber zum Teil auch Ärzte bewegen, pflanzliche Arznei- und Heilmittel in die Therapie und Tertiärprävention mit einzubeziehen sind:

- Subjektive Bedeutung für die Patienten (z.B. individuelle Lebensphilosophie, Einbezug von «natürlichen Therapien» in die eingreifenden Tumortherapien).

- Individuelle Zuordnung von erhofften bzw. zumindest subjektiv notwendigen therapeutischen und präventiven Wirkungen einer Behandlung an die Einnahme phytotherapeutischer Zubereitungen.

- Wissen um das Potential von Phytotherapeutika (lange und offensichtlich sinnvolle sowie nebenwirkungsarme Anwendungstradition, breites Wirkungsspektrum).

- Aufgreifen publizierter, potentiell bei einer Tumorerkrankung therapeutisch nützlicher experimenteller Untersuchungsergebnisse von z.B. bereits lange in anderen Indikationsbereichen therapeutisch verwendeten pflanzlichen Mitteln (als Sonderform einer Art translationalen Ansatzes im Sinne von «from bench to bedside» und «bedside to bench»). Für solche anderweitig bereits klinisch beforschten Phytotherapeutika liegen häufig umfangreiche Daten zur Sicherheit vor.

- Aufgreifen epidemiologischer Hinweise auf möglichen (therapeutischen, primär- und/oder tertiärpräventiven) Nutzen pflanzlicher Zubereitungen aus Ernährung und Phytotherapie.

- Bezug auf individuelle Empirie, Austausch der Erfahrungen unter Mitpatienten und auffällige (publizierte) Kasuistiken.

- Bezug auf Ergebnisse aus Kohortenstudien und kleinen Interventionsstudien.

- Wissenschaftsgestützte Abwägung von möglichem Nutzen und $\mathrm{zu}$ erwartenden Risiken einer (versuchsweisen) Anwendung pflanzlicher Mittel, die mit vergleichbaren Indikationen, wenngleich nicht bei tumorkranken Menschen, bereits seit längerem in therapeutischem Gebrauch bzw. klinisch erforscht sind.

\section{Antiinflammatorische pflanzliche Wirkstoffe und Metastasierung}

Im letzten Jahrzehnt haben chronisch-entzündliche Vorgänge im Zusammenhang mit Tumorentstehung, Tumorausbreitung und insbesondere Metastasenbildung ein stark zunehmendes wissenschaftliches Interesse gefunden [24-37]. Vielfältige chronische Entzündungsvorgänge tragen wesentlich zum Mikroumfeld (supportive tumor-microenvironment, dysregulierte Entzündung) einer möglichen Metastaseninitiation bzw. -ansiedlung bei [24, 26, 28, 30, 31, 33, 36, 38-45]. Sie ermöglichen bzw. erleichtern die Metastasierungschritte. Im übertragenen Sinne wird die Tumorerkrankung z.B. auch als eine Wunde charakterisiert, die nicht heilt $[42,46]$. Die komplexen
Vorgänge einer chronischen Entzündung und einer Beteiligung des angeborenen Immunsystems stehen dabei in enger Wechselwirkung [32, 42, 47]. Eine Dämpfung der verschiedenen Entzündungsreaktionen könnte sich als ein wissenschaftlich begründeter zusätzlicher Bestandteil einer modernen multimodalen Tumortherapie etablieren [24, 39, 48-54]. Eine solche Rolle könnten z.B. immunmodulierend wirkende Mittel spielen, die chronische Entzündungen und dadurch auch Tumorprogression und Metastasierung zu unterdrücken bzw. herabzusetzen vermögen. Zahlreiche pflanzliche Zubereitungen (Einzel- und Kombinationspräparate) aus der westlichen und östlichen Phytotherapie besitzen experimentell, zum Teil auch klinisch nachgewiesene antiinflammatorische und teilweise auch immunmodulierende Wirkungen [2, 10, 11, 29, 39-41, 54-70]. Sie könnten aufgrund dieser Wirkungen z.B. die Angiogenese und Metastasenbildung beeinträchtigen und somit potentiell dem Fortschreiten einer Tumorerkrankung entgegenwirken [29, 30, 40, 41, 54, 55, 70-73].

Die Gesichtspunkte der antiinflammatorisch und gegebenenfalls antitumoral relevanten Untersuchungen werden im Folgenden zusammengefasst:

- Phytotherapeutika und vergleichbare pflanzliche Zubereitungen besitzen als pleiotrope und multimodale Wirkstoffe verschiedene Wirkungen auf Mechanismen, die in Entstehung, Verlauf und Ausbreitung von Tumorerkrankungen involviert sind, so z.B. antikarzinogene, proapoptotische, immunmodulierende und antiinflammatorische Effekte (Multi-Target-Charakter).

- Schwerpunkte der derzeitigen Forschung, aber auch der versuchsweisen Anwendung, beziehen sich auf Angiogenese und Entzündungsvorgänge im Rahmen der Metastasierung (unter anderem Auswirkungen auf den Tumornekrosefaktor (TNF), «nuclear factor kappa-light-chainenhancer of activated $\mathrm{B}$ cells» $\left(\mathrm{NF}_{\mathrm{K}} \mathrm{B}\right)$, Transforming Growth Factor $\beta$ (TGF- $\beta$ ), Cyclooxygenase (COX), Lipoxygenase (LOX), Stickstoffmonoxid (NO), verschiedene Zytokine, Matrix Metalloproteinasen (MMP), Wachstumsfaktor (GF), Toll-like-Rezeptoren).

- Bislang liegen Untersuchungen zu einfachen Drogenzubereitungen (z.B. Drogenpulver), wässrigen Zubereitungen (z.B. Teezubereitungen, Dekokte) und Extrakten mit unterschiedlichen Lösungsmitteln (z.B. mit kombinierten lipo- und hydrophilen Eigenschaften) vor.

- Zudem liegen Untersuchungen zu pflanzlichen Stofffraktionen (phytogene Stoffgemische) aus Arzneidrogen vor. Diese besitzen ebenfalls einen Vielstoffcharakter, z.B. Fraktionen mit polyphenolischem Charakter oder verschiedene Flavonoidfraktionen.

Mittlerweile liegen für Zubereitungen einer zunehmenden Anzahl von Arznei- und Heilpflanzen experimentelle Invitro- bzw. In-vivo-Untersuchungen vor, die vielfältige antientzündliche bzw. antiinflammatorisch relevante Eigenschaften zeigen [24, 34, 35, 46, 51, 52, 64]. Häufig verlaufen antioxidative und antiinflammtorische Effekte parallel [28, 51, 52, 55, 


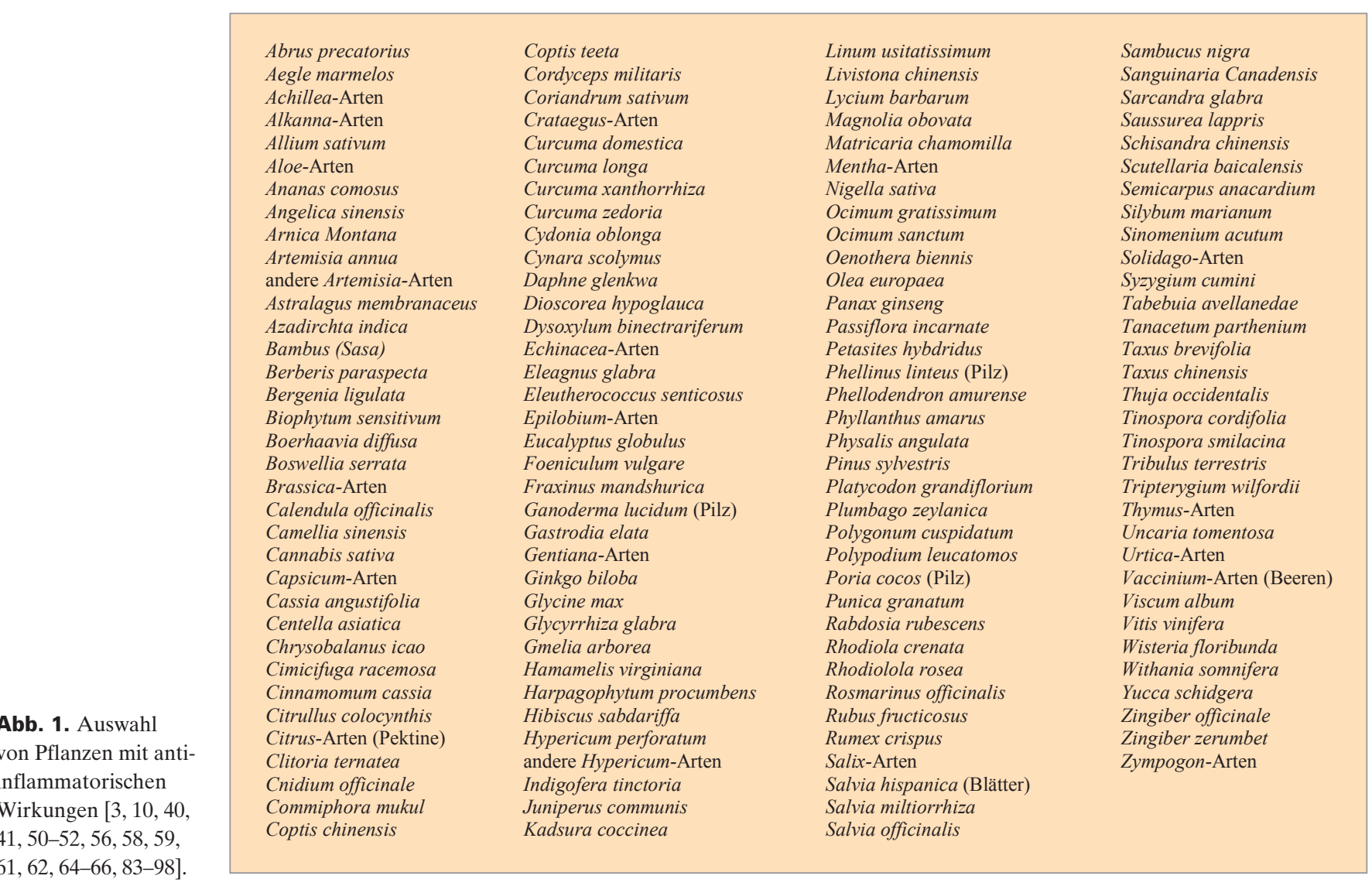

64, 74]. Die Datenlage weist auch für einzelne Drogen und Zubereitungen auf einen potentiellen Nutzen im Zusammenhang mit Tumorausbreitung und Metastasenbildung hin.

Zubereitungen aus wissenschaftlich monographierten Arzneidrogen (z.B. European Scientific Cooperative on Phytotherapy (ESCOP), European Medicines Agency Comittee on Herbal Medicinal Products (EMA-HMPC), World Health Organization (WHO) $[3,11,75-77]$ besitzen im Vergleich zu Zubereitungen aus anderen nichtmonographierten Pflanzen mehrere Vorteile [61]. Aufgrund des Arzneimittelcharakters und der entsprechenden Anforderungen an Qualität und Sicherheit können nachvollziehbare relevante Risikoabschätzungen vorgenommen werden. Ein großer Teil solcher pflanzlicher Produkte weist kein oder nur ein geringes Toxizitätsrisiko auf. Sie können daher auch bei älteren, geschwächten bzw. durch andere Therapien beeinträchtigten und multimorbiden Patienten eingesetzt werden. Zum Teil gelten diese Ausführungen auch für pflanzliche Zubereitungen ohne Arzneimittelcharakter (Supplemente), sofern ihr Gebrauch nachvollziehbar dokumentiert und die Qualität gesichert ist, sowie für ausgewählte Pflanzen in der Ernährung [40, 41, 52, 53, 57, 73, 78-82]. Insgesamt vermögen solche nichtselektiven und multifokal wirkenden pflanzlichen Produkte simultan an unterschiedlichen Orten in die komplexen Vorgänge der Metastasierung einzugreifen [61]. Möglicherweise können diese Vielstoffgemische auch das Risiko der Resistenzentwicklung von Tumorzellen begrenzen [61]. Abbildung 1 zeigt eine beschränkte Auswahl von Pflanzen, für deren Drogen bzw. Zubereitungen experimentell antiinflammatorische bzw. antioxidative Wirkungen und zum Teil weitere Effekte auf andere Schritte im Rahmen von Tumorentstehung und Tumorausbreitung dokumentiert sind.

Zusätzlich zu den Einzeldrogen gibt es zahlreiche Kombinationen von Arznei- und Heilpflanzen aus der europäischen und außereuropäischen Phytotherapie, vor allem aus der TCM, der Kampo-Medizin (Traditionelle Japanische Medizin) und der Ayurvedischen Medizin sowie weiteren ethnomedizinischen Traditionen (z.B. ausgewählte Formula der Traditionellen Tibetischen Medizin), die in einigen europäischen Ländern auch eine Zulassung als Arzneimittel besitzen [56, 59, 60, 63, 65, 99-104].

Arznei- und Heilpflanzen mit ausgeprägten antientzündlichen und antioxidativen Eigenschaften besitzen häufig einen hohen Anteil an polyphenolischen Komponenten [2, 11, 14, 15, 54, 73]. Vergleichbar ist die Situation bei antioxidativ wirkenden pflanzlichen Lebensmitteln [53, 57, 78, 79, 105]. Wenigstens zum Teil sind dabei die intrazellulär für eine Beeinflussung der Signaltransduktion erforderlichen Konzentrationen deutlich niedriger als diejenigen, die für eine Beeinflussung der antioxidativen Kapazität notwendig wären [57]. Auch andere Wirkstoffkomponenten wie Terpene weisen auf antientzündliche Wirkungen hin [14, 15, 47]. 
Ocimum gratissimum, eine wichtige Arzneipflanze aus der TCM, ist eine prototypische Pflanze, für die neben antiinflammatorischen Wirkungen auch weitere antitumorale Effekte dokumentiert sind [106], die bei tumorkranken Menschen nützlich sein könnten (Hemmung des Tumorwachstums, Abnahme der Angiogenese). Zudem zeigen Untersuchungen, dass das Vielstoffgemisch (Blattextrakt) sich als wirksamer erwies, als seine vermeintlichen Hauptwirkungsträger (die Monosubstanzen Eugenol, Apigenin, Ursolsäure) allein [106]. Auch für eine Reihe weiterer Pflanzen, die aus unterschiedlichen, zumeist supportiven Gründen bei tumorkranken Menschen eingesetzt werden, liegen mittlerweile Untersuchungen vor, die zeigen, dass die phytogenen Vielstoffgemische wirksamer sind als die vermeintlichen Wirkungsträger mit Monosubstanzcharakter, z.B. Zubereitungen aus Johanniskraut (Extrakt mit Arzneimittelcharakter; vermeintlicher Wirkungsträger Hyperforin) [19, 107], Zubereitungen aus Weidenrinde (Extrakt mit Arzneimittelcharakter; vermeintlicher Wirkungsträger Salicylverbindungen) [108] oder Zubereitungen aus dem Indischen Weihrauch (standardisierter Extrakt; vermeintlicher Wirkungsträger: Boswelliasäuren) [109]. Zingiber officinale, Ginkgo biloba, CurcumaArten und Artemisia annua sind antiinflammatorisch wirksame Arznei- bzw. Heilpflanzen, deren Drogen und Zubereitungen bereits eine lange Anwendungstradition außerhalb der Behandlung tumorkranker Menschen besitzen. Experimentelle Untersuchungen weisen darauf hin, dass sich ihre Anwendung auch im Zusammenhang mit Tumorerkrankungen als nützlich erweisen könnte [41, 55, 62, 86, 88, 94]. Die vorliegenden langjährigen therapeutischen Erfahrungen und Studien außerhalb der Onkologie [11] könnten eine tragfähige Basis für die versuchsweise Anwendung im Rahmen einer antitumoral ausgerichteten Therapie bieten.

Untersuchungen über definierte vielfältige antientzündliche Eigenschaften und weitere Tumor hemmende Wirkungen liegen auch für sehr komplexe Mischungen der TCM und anderer ethnomedizinischer Systeme vor [63]. Ein solches Beispiel ist das aus der Kampo-Medizin und der TCM stammende und in Japan bei tumorkranken Menschen häufig angewendete Kombinationspräparat Juzen-Taiho-To [110], das aus 10 Drogen besteht (Pilzdrogen werden formal der Phytotherapie zugeordnet): Zimtrinde (Cinnamomum cassia), Süßholzwurzel (Glycyrrhiza glabra und Glycyrrhiza uralensis), Japanische Engelwurzwurzel (Angelica acutiloba, Angelica acutiloba var. sugiyamae), Astragaluswurzel (Astragalus mongholicus oder Astragalus membranaceus), Atractylodeswurzel (Atractylodes lancea und Atractylodes chinensis) und Ginsengwurzel (Panax ginseng) sowie als weitere Bestandteile Päonienwurzel (Paeonia lactiflora), Rehmanniawurzel (Braunwurz (Rehmannia glutinosa)), Cnidiumwurzel (Brenndolde (Cnidium officinale)) und Indischer Brotpilz (Poria cocos). Relativ umfangreiche experimentelle Untersuchungen (in vitro, in vivo) zeigen potentiell relevante antitumorale Wirkungen im Zusammenhang mit Karzinogenese, Tumor- progression sowie Metastasierung [110]. Vergleichbare Hinweise finden sich auch für andere komplexe pflanzliche Mischungen der TCM [111]. Die relativ einfache antioxidativ und antiinflammatorisch wirksame Ayurvedische Formel «Triphala», eine Kombination aus jeweils dem Perikarp von Phyllanthus emblica (Emblica officinalis), Terminalia chebula und Terminalia belerica weist ein breites Spektrum von Wirkungen auf, die bei tumorkranken Menschen nützlich sein könnten - chemopräventive, radioprotektive, antimutagene, antiklastogene und immunmodulierende Effekte mit pleiotropen Wirkmechnismen [60]. Pleiotrope antientzündliche Wirkungen sind experimentell auch für das tibetische Multikomponentenpräparat «PADMA-28» dokumentiert (enthält unter anderem 21 pflanzliche Drogen) [99-102]. Unterstützend könnten therapierelevante Daten (Sicherheit, unerwünschte Wirkungen) von einem in klinischen Studien geprüften anderen Indikationsbereich mit herangezogen werden [112].

Vereinzelt wurden solche antiinflammatorisch ausgerichteten Ansätze bislang in klinischen Studienansätzen überprüft. In einer Kohortenstudie an über 200 Patienten mit kolorektalen Karzinomen unter kurativ intendierter konventioneller (westlicher) Tumortherapie (einschließlich Operation, Chemotherapie und/oder Radiotherapie) wurde der mögliche Einfluss einer zusätzlichen Behandlung mit einer phytotherapeutischen chinesischen Arzneimitteltherapie untersucht. Die Hälfte der Patienten erhielt zusätzlich zur konventionellen Tumortherapie eine individuell zusammengesetzte, an den jeweiligen Symptomen ausgerichtete orale TCM-Therapie, die andere Hälfte nur die konventionelle Krebsbehandlung [68]. Für die individuelle Auswahl der Phytotherapeutika waren die jeweils spezifischen Beschwerden des einzelnen Patienten ausschlaggebend. Mit der zusätzlichen TCMBehandlung sollte das Potential von pflanzlichen Mitteln bzw. phytotherapeutischen Kombinationen geprüft werden, neben den symptomatischen Einflüssen auch die Tumorerkrankung selbst zu beeinflussen (Tab. 3).

Einfache bzw. komplexe phytotherapeutische Behandlungen können neben unterschiedlichen, z.B. symptombezogenen spezifischen Wirkungen, auch gemeinsame Gruppeneffekte besitzen, z.B. antiinflammatorische Eigenschaften. Mit solchen Effekten ermöglichen die phytotherapeutischen Vielstoffgemische das gleichzeitige Ausnutzen von Arzneimittel übergreifenden «Systemeigenschaften» (z.B. Antiinflammation) und arzneimittelspezifischen, beschwerdeorientierten weiteren Wirkungen (z.B. Behandlung von Übelkeit oder Müdigkeit und Erschöpfung). Nicht jeder Patient, der antiinflammatorisch behandelt werden sollte, braucht das gleiche Phytotherapeutikum zu erhalten, denn antiinflammatorische Eigenschaften kommen, bei ansonsten unterschiedlichen Wirkungen, bei verschiedenen pflanzlichen Mitteln in vergleichbarem Ausmaß vor. Aus einem solchen Therapieansatz kann sich für Patienten die Option ergeben, neben der Linderung von Beschwerden auch antitumorale Wirkungen zu erfahren. 
Tab. 3. Beispiel einer symptomorientierten Auswahl antientzündlich wirkender pflanzlicher Mittel Ergebnisse [68]

\begin{tabular}{|c|c|c|c|c|}
\hline \multirow[t]{3}{*}{ Kohorten } & \multicolumn{4}{|c|}{ Relaps/Metastasierung } \\
\hline & \multicolumn{2}{|c|}{$\begin{array}{l}\text { «westliche» (konventionelle) Behandlung } \\
(\mathrm{N}=104)^{\mathrm{b}}\end{array}$} & \multicolumn{2}{|c|}{ Kombinationsbehandlung $(\mathrm{N}=98)^{\mathrm{c}}$} \\
\hline & $\mathrm{n}$ & $\%$ & $\mathrm{n}$ & $\%$ \\
\hline $1 \mathrm{Jahr}$ & $5 / 104$ & 4,8 & $0 / 98$ & 0 \\
\hline 2 Jahre & $17 / 104$ & 16,4 & $2 / 98$ & 2,0 \\
\hline 3 Jahre & $21 / 97$ & 21,7 & $9 / 77$ & 11,7 \\
\hline 4 Jahre & $21 / 81$ & 25,9 & $9 / 64$ & 14,1 \\
\hline 5 Jahre & $21 / 55$ & 38,2 & $10 / 47$ & 21,3 \\
\hline \multicolumn{5}{|c|}{ 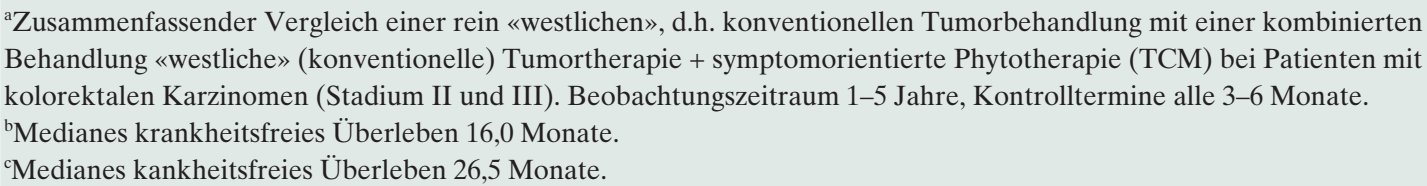 } \\
\hline
\end{tabular}

Klinisch-epidemiologische Hinweise auf eine potentielle primär- bzw. tertiär-chemopräventive Wirksamkeit phytogener Gemische zeigten sich in einer allerdings relativ kleinen Kohortenstudie ( $\mathrm{n}=87 ; 36$ Patienten mit reseziertem Kolonkarzinom und 51 Patienten nach Polypektomie; Beobachtungsdauer 3-4 Jahre; Kontrolle $\mathrm{n}=56$ nach Matching vergleichbarer Patienten), die ein Gemisch aus Catechinen (standardisiert auf Epigallocatechingallat aus grünem Tee) und Flavonoiden (standardisiert auf Apigenin) mit jeweils 20 mg/Tag erhalten hatten [80]. Bei den behandelten Patienten entwickelte sich im Gegensatz zu den Kontrollpatienten kein Karzinom, zudem traten weniger Polypen auf. Neben anderen tumorrelevanten Wirkungen besitzt dieses Gemisch, ebenso wie vergleichbare Wirkstoffkombinationen, ausgeprägte antientzündliche Eigenschaften [73].

Solch relativ niedrige interventionell verabreichte Flavonoidmengen lassen sich auch mit regelmäßiger Einnahme einer ganzen Reihe von häufig verwendeten Phytotherapeutika in üblicher Dosierung erreichen. So enthält z.B. Johanniskraut 20-40 mg Flavonoide/g Droge, die durchschnittliche therapeutische Drogenmenge pro Tag liegt bei $4 \mathrm{~g}[2,11]$. Der Multikomponentenwirkstoff «Johanniskraut» weist experimentell vielfältige Wirkungen auf, deren Ausnutzung sich bei tumorkranken Menschen als sinnvoll erweisen könnte (breitgefächerte antientzündliche und antioxidative Wirkungen, wundheilungsfördernde Eigenschaften, proapoptotische Effekte in verschiedenen Tumormodellen) [11, 13, 16, 19, 107, 113-117]. Unter symptomorientierten Gesichtspunkten könnte sich Johanniskraut mit seinen experimentell definierten antientzündlichen Wirkungen gerade bei tumorkranken Patienten mit depressiven Verstimmungen als vorteilhaft erweisen. Selbstverständlich muss das mögliche Interaktionsrisiko berücksichtigt werden, wenn Patienten weitere Arzneimittel einnehmen müssen. Bei gleichzeitigen weiteren Arzneimittelbehandlungen sollte ein Johanniskrautpräparat mit einem niedrigen Interaktionspotential gewählt werden. Zur
Frage der klinischen Anwendung bei tumorkranken Menschen lassen sich, wenngleich mit Einschränkungen, die umfangreichen Daten aus klinischen Studien in anderen Anwendungsbereichen heranziehen [118-120]. Vergleichbare Überlegungen könnten bei anderen potentiell antientzündlich wirkenden Arzneidrogen und ihren Zubereitungen zum Tragen kommen.

Grüner Tee von guter Qualität enthält bei ausreichender Dosierung sowohl ausreichend Epigallocatechingallat (etwa $160 \mathrm{mg} / 2,5 \mathrm{~g}$ ) wie auch bedeutsame Mengen an verschiedenen Flavonoiden $(20 \mathrm{mg} / 2,5 \mathrm{~g})$ [2, 11, 16]. So lässt sich z.B. grüner Tee (Quelle für Epigallocatechingallat) mit einer symptomatisch ausgewählten weiteren flavonoidreichen Arzneidroge kombinieren. Zubereitungen aus Camellia sinensis (Teeblätter), vor allem mit einem hohen Anteil an Catechinen wie Epigallocatechingallat, besitzen neben ausgeprägten antiinflammatorischen Wirkungen weitere vielfältige Effekte auf Tumorentstehung und Tumorausbreitung [66, 121].

Vergleichbar mit dem Johanniskraut lassen sich auch andere Arzneidrogen mit Gruppeneigenschaften und zusätzlichen speziellen Wirkungen zusammenfassen. Eine für viele tumorkranke Menschen wichtige Droge sind die Früchte der Mariendistel (Silybum marianum) und deren Spezialextrakte, die hochkonzentriert vor allem das Flavonoidgemisch Silymarin enthalten [2,11]. Für den Spezialextrakt liegen mittlerweile zahlreiche experimentelle Untersuchungen vor, die auf vielfältige antientzündliche Wirkungen (Pleiotropie) auf den verschiedenen Ebenen des Entzündungsgeschehens hinweisen $[11,21,61,122]$. Zudem ließen sich mittlerweile zusätzliche potentiell Tumor hemmende Wirkungen finden [61, 71, 122-124]. Neue experimentelle Forschungen zeigen weitere nicht direkt antitumorale Effekte, die bei tumorkranken Menschen sinnvoll sein könnten, z.B. im Zusammenhang mit Chemotherapien und Radiotherapien sowie deren Nebenwirkungen [21, 122-125]. Natürlich könnten solche antientzündlichen Wirkungen nicht nur bei Tumorerkrankungen nützlich 
sein, sondern auch bei einer Reihe weiterer Erkrankungen, bei denen chronische Entzündungsvorgänge eine wesentliche Rolle spielen [122]. Im Hinblick auf mögliche unerwünschte Wirkungen lassen sich bedingt Daten aus klinischen Untersuchungen in anderen Anwendungsbereichen heranziehen [126, 127].

Die Eigenschaften pflanzlicher Mittel im Zusammenhang mit Entzündungen und Entzündungshemmung und deren potentielle Auswirkungen spielen auch, wenngleich sehr vereinfacht und teilweise widersprüchlich, in Betrachtungsweisen der TCM eine Rolle [43, 128, 129]. So werden z.B. proinflammatorische Abläufe (unter anderem Apoptose) als «Yin» und postinflammatorische Abläufe (unter anderem antiinflammatorische Einflüsse, Wundheilung, Gewebsreparatur) als «Yang» charakterisiert [129].

\section{Schlussfolgerung}

Zusammenfassend kann in einer relevanten Anzahl phytotherapeutischer Arznei- und Heilmittel, die in verschiedenen Medizintraditionen supportiv in der Behandlung von Tumorpatienten angewendet werden, das Potential für antitumoral relevante Wirkmechanismen gesehen werden. Die pflanzlichen Wirkstoffe sind Vielstoffgemische mit Multi-TargetEigenschaften und pleiotropen antiinflammatorischen Wir- kungen. Diese könnten in der Verhinderung bzw. Verzögerung der Metastasierung eine bedeutsame Rolle spielen. Hierzu liegt mittlerweile eine große Anzahl experimenteller Daten vor. Wenngleich bislang nur wenige klinische Untersuchungen veröffentlicht sind, so könnten Behandlungsversuche dennoch nützlich und vertretbar erscheinen, sofern für solche Arznei- und Heilpflanzen gesicherte Daten über Qualität und Sicherheit dokumentiert sind und eine Übertragung auf die Situation tumorkranker Menschen nach fachlich fundierter und kritischer Abwägung möglich erscheint. Zahlreiche der in der Supportivtherapie eingesetzten Phytotherapeutika besitzen neben ihrer symptomatischen Wirksamkeit auch antiinflammatorische Wirkungen. Die Auswahl von supportiv wirksamen Phytotherapeutika mit zusätzlichen antiinflammatorischen Effekten könnte darüber hinaus die Möglichkeit einer antitumoralen Wirksamkeit bieten. Das therapeutische Potential wird heute erst in Ansätzen gesehen und kann klinisch aber bei Weitem noch nicht abschließend eingeschätzt werden. Insgesamt besteht ein großer Bedarf an sinnvoll geplanter und therapeutisch orientierter Forschung.

\section{Disclosure Statement}

Die Autoren erklären im Bezug auf diese Arbeit in keinem Interessenskonflikt zu stehen.

\section{Literatur}

1 Saller R, Reichling J: Phytotherapie; in Melchart D, Brenke R, Dobos G, Gaisbauer M, Saller R (Hrsg): Naturheilverfahren - Leitfaden für die ärztliche Aus-, Fort- und Weiterbildung. Stuttgart, Schattauer, 2002, pp 180-230.

2 Saller R, Reichling J, Hellenbrecht D: Phytotherapie - klinische, pharmakologische und pharmazeutische Grundlagen. Heidelberg, Haug, 1995.

3 World Health Organization (WHO) (ed): WHO Monographs on Selected Medicinal Plants. Geneva, World Health Organization, 1999-2007.

4 Schmitz R: Geschichte der Pharmazie, Band 1. Von den Anfängen bis zum Ausgang des Mittelalters. Eschborn, Govi-Verlag, 1998.

5 Delaveau P: Histoire et renouveau des plantes médicinales. Paris, Albin Michel, 1982.

6 Pavord A: Wie die Pflanzen zu ihren Namen kamen. Eine Kulturgeschichte der Botanik. Berlin, Berlin Verlag, 2008.

7 Bendum J, Loew D, Schilcher H: Arzneipflanzen in der traditionellen Medizin, ed 2. Bonn, Kooperation Phytopharmaka, 1994.

8 Mayer J, Uehleke B, Saum K: Handbuch der Klosterheilkunde, ed 6. München, Zabert Sandmann, 2003.

9 Strank K, Meures-Balke J: Obst, Gemüse und Kräuter Karls des Großen. Mainz, Philipp von Zabern, 2008.

10 Jiang M, Yang J, Zhang C, Liu B, Chan K, Cao $\mathrm{H}, \mathrm{Lu} \mathrm{A}$ : Clinical studies with traditional Chinese medicine in the past decade and future research and development. Planta Med 2010;76:2048-2064.
11 Blaschek W, Hilgenfeldt U, Holzgrabe U, Reichling R, Ruth P, Schulz V (eds): HagerROM 2010. Hagers Enzyklopädie der Arzneistoffe und Drogen. Heidelberg, Springer, 2010.

12 Saller R, Melzer J: Arznei- und Heilpflanzen: Ein abgestuftes und globales Arzneimittelangebot. Forsch Komplementärmed Klass Naturheilkd 2004;11:264-266.

13 Saller R, Melzer J, Reichling J: Johanniskraut ( $H y$ pericum perforatum): Ein plurivalenter Rohstoff für traditionelle und moderne Therapien. Forsch Komplementärmed Klass Naturheilkd 2003;10 (suppl 1):33-40.

14 Wink M: Wirkungen von in der Phytotherapie eingesetzten Vielkomponenten-Gemischen auf Proteine, Gene und Biomembranen. Schweiz Zschr Ganzheits Med 2009;21:42-53.

15 Wink M: Evolutionary advantage and molecular modes of action of multi-component mixtures used in phytomedicine. Curr Drug Metab 2008;9:9961009.

16 Reichling J, Hostanska K, Saller R: St. John's wort (Hypericum perforatum l.) - multicompound preparations versus single substances. Forsch Komplementärmed Klass Naturheilkd 2003;10(suppl 1):28-32.

17 Saller R, Holzer B: Multimorbiditat, Komorbidität und phytotherapeutische Vielstoffgemische als Arzneimittel. Forsch Komplementmed 2010;17:300-302.

18 Shay NF, Banz WJ: Regulation of gene transcription by botanicals: novel regulatory mechanisms. Annu Rev Nutr 2005;25:297-315.
19 Dell'Aica I, Caniato R, Biggin S, Garbisa S: Matrix proteases, green tea, and St. John's wort: biomedical research catches up with folk medicine. Clin Chim Acta 2007;381:69-77.

20 Saller R, Melzer J, Uehleke B, Rostock M: Phytotherapeutische Bittermittel. Schweiz Zschr Ganzheits Med 2009;21:200-205.

21 Post-White J, Ladas EJ, Kelly KM: Advances in the use of milk thistle (Silybum marianum). Integr Cancer Ther 2007;6:104-109.

22 Saller R, Reichling J, Rostock M, Iten F, Melzer $\mathrm{J}$ : Gastrointestinal symptoms in cancer patients possible phytotherapy approaches. Schweiz Zschr Ganzheits Med 2008;20:221-230.

23 Melzer J, Iten F, Hostanska K, Saller R: Efficacy and safety of mistletoe preparations (viscum album) for patients with cancer diseases. A systematic review. Forsch Komplementmed 2009;16:217226.

24 Luqman S, Pezzuto JM: NFkappaB: a promising target for natural products in cancer chemoprevention. Phytother Res 2010;24:949-963.

25 Muller-Hubenthal B, Azemar M, Lorenzen D, Huber M, Freudenberg MA, Galanos C, Unger C, Hildenbrand B: Tumour biology: tumour-associated inflammation versus antitumor immunity. Anticancer Res 2009:29:4795-4805.

26 Wallace A, Gibson D, Saunders P, Jabbour HN: Inflammatory events in endometrial adenocarcinoma. J Endocrinol 2010;206:141-157.

27 Ralhan R, Pandey MK, Aggarwal BB: Nuclear factor-kappaB links carcinogenic and chemopreventive agents. Front Biosci (Schol Ed) 2009;1:45-60. 
28 Chatterjee S, Fisher AB: Free radicals, oxidative stress, and cancer; in Bagchi D, Preuss HG (eds): Phytopharmaceuticals in Cancer Chemoprevention. Boca Raton, CRC Press, 2005, pp 171-186.

29 Fan TP, Yeh JC, Leung KW, Yue PY, Wong RN: Angiogenesis: from plants to blood vessels. Trends Pharmacol Sci 2006;27:297-309.

30 Mantovani A, Garlanda C, Allavena P: Molecular pathways and targets in cancer-related inflammation. Ann Med 2010;42:161-170.

31 Moore MM, Chua W, Charles KA, Clarke SJ: Inflammation and cancer: causes and consequences. Clin Pharmacol Ther 2010;87:504-508.

32 Terzic J, Grivennikov S, Karin E, Karin M: Inflammation and colon cancer. Gastroenterology 2010; 138:2101-2114.

-33 Wu Y, Zhou BP: Inflammation: a driving force speeds cancer metastasis. Cell Cycle 2009;8:32673273.

34 Aggarwal BB, Vijayalekshmi RV, Sung B: Targeting inflammatory pathways for prevention and therapy of cancer: short-term friend, long-term foe. Clin Cancer Res 2009:15:425-430.

-35 Balkwill F, Charles KA, Mantovani A: Smoldering and polarized inflammation in the initiation and promotion of malignant disease. Cancer Cell 2005; 7:211-217.

-36 Solinas G, Marchesi F, Garlanda C, Mantovani A, Allavena P: Inflammation-mediated promotion of invasion and metastasis. Cancer Metastasis Rev 2010;29:243-248.

\37 Yoneda T, Hata K, Nakanishi M, Nagae M, Nagayama T, Wakabayashi H, Nishisho T, Sakurai T, Hiraga T: Involvement of acidic microenvironment in the pathophysiology of cancer-associated bone pain. Bone 2011;48:100-105.

38 Balkwill F, Mantovani A: Cancer and inflammation: implications for pharmacology and therapeutics. Clin Pharmacol Ther 2010;87:401-406.

39 Kale A, Gawande S, Kotwal S: Cancer phytotherapeutics: role for flavonoids at the cellular level. Phytother Res 2008;22:567-577.

40 Sagar SM, Yance D, Wong RK: Natural health products that inhibit angiogenesis: a potential source for investigational new agents to treat cancer - part 2. Curr Oncol 2006;13:99-107.

41 Sagar SM, Yance D, Wong RK: Natural health products that inhibit angiogenesis: a potentia source for investigational new agents to treat cancer - part 1. Curr Oncol 2006;13:14-26.

42 Stix G: Bösartige Entzündungen. Spekt Wiss 2008; 3:50-57.

43 Danese S, Mantovani A: Inflammatory bowel disease and intestinal cancer: a paradigm of the yinyang interplay between inflammation and cancer. Oncogene 2010;29:3313-3323.

44 Lawrence T: Inflammation and cancer: a failure of resolution? Trends Pharmacol Sci 2007;28:162-165.

45 Ioannou S, Voulgarelis M: Toll-like receptors, tissue injury, and tumourigenesis. Mediators Inflamm 2010; doi: $10.1155 / 2010 / 581837$.

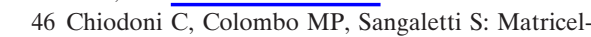
lular proteins: from homeostasis to inflammation, cancer, and metastasis. Cancer Metastasis Rev 2010; 29:295-307.

47 Rios JL: Effects of triterpenes on the immune system. J Ethnopharmacol 2010;128:1-14

48 Patwardhan B, Vaidya AD: Natural products drug discovery: accelerating the clinical candidate development using reverse pharmacology approaches. Indian J Exp Biol 2010;48:220-227.

49 Paul AT, Gohil VM, Bhutani KK: Modulating TNF-alpha signaling with natural products. Drug Discov Today 2006;11:725-732.
50 Darshan S, Doreswamy R: Patented antiinflammatory plant drug development from traditional medicine. Phytother Res 2004;18:343-357.

51 Aravindaram K, Yang NS: Anti-inflammatory plant natural products for cancer therapy. Planta Med 2010;76:1103-1117.

52 Amin AR, Kucuk O, Khuri FR, Shin DM: Perspectives for cancer prevention with natural compounds. J Clin Oncol 2009;27:2712-2725.

53 Thomasset S, Teller N, Cai H, Marko D, Berry DP, Steward WP, Gescher AJ: Do anthocyanins and anthocyanidins, cancer chemopreventive pigments in the diet, merit development as potential drugs? Cancer Chemother Pharmacol 2009;64:201-211.

54 Prasad S, Phromnoi K, Yadav VR, Chaturvedi MM, Aggarwal BB: Targeting inflammatory pathways by flavonoids for prevention and treatment of cancer. Planta Med 2010;76:1044-1063.

55 Yance DR Jr., Sagar SM: Targeting angiogenesis with integrative cancer therapies. Integr Cancer Ther 2006;5:9-29.

56 You HQ, Rafi MM, Ho CT: Targeting inflammation using asian herbs; in Ho CT (ed): Herbs: Challenges in Chemistry and Biology. Oxford, Oxford University Press, 2006, pp 266-280.

57 Crozier A, Jaganath IB, Clifford MN: Dietary phenolics: chemistry, bioavailability and effects on health. Nat Prod Rep 2009;26:1001-1043.

58 Dinarello CA: Anti-inflammatory agents: present and future. Cell 2010;140:935-950.

59 Parekh HS, Liu G, Wei MQ: A new dawn for the use of traditional Chinese medicine in cancer therapy. Mol Cancer 2009;8:21.

60 Baliga MS: Triphala, Ayurvedic formulation for treating and preventing cancer: a review. J Altern Complement Med 2010;16:1301-1308.

61 Deep G, Agarwal R: Antimetastatic efficacy of silibinin: molecular mechanisms and therapeutic potential against cancer. Cancer Metastasis Rev 2010;29:447-463.

62 Ferreira JF, Luthria DL, Sasaki T, Heyerick A: Flavonoids from Artemisia annua l. as antioxidants and their potential synergism with artemisinin against malaria and cancer. Molecules 2010;15: 3135-3170.

63 Garodia P, Ichikawa H, Malani N, Sethi G, Aggarwal BB: From ancient medicine to modern medicine: Ayurvedic concepts of health and their role in inflammation and cancer. J Soc Integr Oncol 2007;5:25-37.

64 Gol'dberg ED, Amosova EN, Zueva EP, Razina TG, Krylova SG, Reikhart DV: Effects of extracts from medicinal plants on the development of metastatic process. Bull Exp Biol Med 2004;138:288294.

65 Ichikawa H, Nakamura Y, Kashiwada Y, Aggarwal BB: Anticancer drugs designed by mother nature: ancient drugs but modern targets. Curr Pharm Des 2007;13:3400-3416.

66 Pajonk F, Riedisser A, Henke M, McBride WH, Fiebich B: The effects of tea extracts on proinflammatory signaling. BMC Med 2006;4:28.

67 Qi F, Li A, Inagaki Y, Gao J, Li J, Kokudo N, Li $\mathrm{XK}$, Tang W: Chinese herbal medicines as adjuvant treatment during chemo- or radio-therapy for cancer. Biosci Trends 2010;4:297-307.

68 Yang YF, Ge JZ, Wu Y, Xu Y, Liang BY, Luo L, Wu XW, Liu DQ, Zhang X, Song FX, Geng ZY: Cohort study on the effect of a combined treatment of traditional Chinese medicine and Western medicine on the relapse and metastasis of 222 patients with stage ii and iii colorectal cancer after radical operation. Chin J Integr Med 2008;14:251-256.
69 Reichling J, Schnitzler P, Suschke U, Saller R: Essential oils of aromatic plants with antibacterial, antifungal, antiviral, and cytotoxic properties - an overview. Forsch Komplementmed 2009;16:79-90.

70 Shu L, Cheung KL, Khor TO, Chen C, Kong AN: Phytochemicals: cancer chemoprevention and suppression of tumor onset and metastasis. Cancer Metastasis Rev 2010;29:483-502.

71 McCarty MF, Block KI: Multifocal angiostatic therapy: an update. Integr Cancer Ther 2005;4:301314.

72 Naldini A, Carraro F: Role of inflammatory mediators in angiogenesis. Curr Drug Targets Inflamm Allergy 2005;4:3-8.

73 Hoensch HP, Kirch W: Potential role of flavonoids in the prevention of intestinal neoplasia: a review of their mode of action and their clinical perspectives. Int J Gastrointest Cancer 2005;35:187-195.

74 Mazzio EA, Soliman KF: In vitro screening for the tumoricidal properties of international medicinal herbs. Phytother Res 2009;23:385-398.

75 European-Medicines-Agency: European Medicines Agency. Herbal medicines for human use. www.ema.europa.eu/ema/index.jsp? curl=/pages/ medicines/landing/herbal_search.jsp\&murl=menus/ medicines/medicines. $j$ sp \& mid $=$ WC0b01ac058001 fa1d\&jsenabled=true (accessed July 19, 2011).

76 European-Scientific-Cooperative-on-Phytotherapy: ESCOP Monographs. The Scietific Foundation for Herbal Medicinal Products., ed 2, Stuttgart, Thieme, 2003

77 European-Scientific-Cooperative-on-Phytotherapy: ESCOP Monographs. The Scientific Foundation for Herbal Medicinal Products. ed 2, Supplement 2009, Stuttgart, Thieme 2009.

78 Aura A: Microbial metabolism of dietary phenolic compounds in the colon. Phytochem Rev 2008;7: 407-429.

79 Wang L, Lee IM, Zhang SM, Blumberg JB, Buring JE, Sesso HD: Dietary intake of selected flavonols, flavones, and flavonoid-rich foods and risk of cancer in middle-aged and older women. Am J Clin Nutr 2009;89:905-912.

80 Hoensch H, Groh B, Edler L, Kirch W: Prospective cohort comparison of flavonoid treatment in patients with resected colorectal cancer to prevent recurrence. World J Gastroenterol 2008;14:21872193.

81 Gates MA, Tworoger SS, Hecht JL, De Vivo I, Rosner B, Hankinson SE: a prospective study of dietary flavonoid intake and incidence of epithelial ovarian cancer. Int J Cancer 2007;121:2225-2232.

82 Chen C, Kong AN: Dietary cancer-chemopreventive compounds: from signaling and gene expression to pharmacological effects. Trends Pharmacol Sci 2005;26:318-326.

83 Tu Y, Roberts L, Shetty K, Schneider SS: Rhodiola crenulata induces death and inhibits growth of breast cancer cell lines. J Med Food 2008;11:413-423.

84 Omer B, Krebs S, Omer H, Noor TO: Steroid-sparing effect of wormwood (Artemisia absinthium) in Crohn's disease: a double-blind placebo-controlled study. Phytomedicine 2007;14:87-95.

85 Nassiri-Asl M, Hosseinzadeh H: Review of the pharmacological effects of Vitis vinifera (grape) and its bioactive compounds. Phytother Res 2009;23: 1197-1204.

86 Mahadevan S, Park Y: Multifaceted therapeutic benefits of Ginkgo biloba l.: chemistry, efficacy, safety, and uses. J Food Sci 2008;73:R14-19.

87 Krebs S, Omer TN, Omer B: Wormwood (Artemisia absinthium) suppresses tumour necrosis factor alpha and accelerates healing in patients with Crohn's disease - a controlled clinical trial. Phytomedicine 2010;17:305-309. 
88 Krishna S, Bustamante L, Haynes RK, Staines HM: Artemisinins: their growing importance in medicine. Trends Pharmacol Sci 2008;29:520-527.

-89 Gogtay NJ, Bhatt HA, Dalvi SS, Kshirsagar NA: The use and safety of non-allopathic indian medicines. Drug Saf 2002;25:1005-1019.

90 Blaschek W, Ebel S, Hilgenfeldt U, Holzgrabe U, Reichling R, Schulz V (eds): HagerROM 2008. Hagers Enzyklopädie der Arzneistoffe und Drogen. Heidelberg, Springer, 2008.

91 Van Slambrouck S, Daniels AL, Hooten CJ, Brock SL, Jenkins AR, Ogasawara MA, Baker JM, Adkins G, Elias EM, Agustin VJ, Constantine SR, Pullin MJ, Shors ST, Kornienko A, Steelant WF: Effects of crude aqueous medicinal plant extracts on growth and invasion of breast cancer cells. Oncol Rep 2007;17:1487-1492.

92 Shukla Y, Singh M: Cancer preventive properties of ginger: a brief review. Food Chem Toxicol 2007; 45:683-690.

93 Hseu YC, Wu CR, Chang HW, Kumar KJ, Lin MK, Chen CS, Cho HJ, Huang CY, Lee HZ, Hsieh WT, Chung JG, Wang HM, Yang HL: Inhibitory effects of Physalis angulata on tumor metastasis and angiogenesis. J Ethnopharmacol 2011;135:762-771.

94 Efferth T, Li PC, Konkimalla VS, Kaina B: From traditional Chinese medicine to rational cancer therapy. Trends Mol Med 2007;13:353-361.

95 Mukherjee PK, Kumar V, Kumar NS, Heinrich M: The Ayurvedic medicine Clitoria ternatea from traditional use to scientific assessment. J Ethnopharmacol 2008;120:291-301.

96 Miller SC: Echinacea: a miracle herb against aging and cancer? Evidence in vivo in mice. Evid Based Complement Alternat Med 2005;2:309-314.

97 Ichikawa H, Takada Y, Shishodia S, Jayaprakasam B, Nair MG, Aggarwal BB: Withanolides potentiate apoptosis, inhibit invasion, and abolish osteoclastogenesis through suppression of nuclear factor-kappaB (NF-kappaB) activation and NFkappaB-regulated gene expression. Mol Cancer Ther 2006;5:1434-1445.

98 Kirste S, Treier M, Wehrle SJ, Becker G, AbdelTawab M, Gerbeth K, Hug MJ, Lubrich B, Grosu AL, Momm F: Boswellia serrata acts on cerebral edema in patients irradiated for brain tumors: a prospective, randomized, placebo-controlled, double-blind pilot trial. Cancer 2011. doi: $10.1002 /$ cncr.25945.

>99 Exner M, Raith M, Holzer G, Gmeiner B, Wagner O, Kapiotis S: Anti-inflammatory mechanisms of the Tibetan herbal preparation Padma 28 in the vessel wall. Forsch Komplementmed 2006;13(suppl 1):13-17.

-100 Navab R, Aingorn H, Fallavollita L, Sallon S, Mechoulam R, Ginsburg I, Vlodavsky I, Brodt P PADMA-28, a traditional Tibetan herbal preparation, blocks cellular responses to bFGF and IGF-I. Inflammopharmacology 2004;12:373-389.

101 Barak V, Kalickman I, Halperin T, Birkenfeld S, Ginsburg I: PADMA-28, a Tibetan herbal preparation is an inhibitor of inflammatory cytokine production. Eur Cytokine Netw 2004;15:203-209.
102 Jenny M, Schwaiger W, Bernhard D, Wrulich OA, Cosaceanu D, Fuchs D, Ueberall F: Apoptosis induced by the Tibetan herbal remedy PADMA 28 in the T cell-derived lymphocytic leukaemia cell line CEM-C7H2. J Carcinog 2005;4:15.

103 Suter M, Richter C: Anti- and pro-oxidative properties of PADMA 28, a Tibetan herbal formulation. Redox Rep 2000;5:17-22.

104 Aggarwal BB, Ichikawa H, Garodia P, Weerasinghe P, Sethi G, Bhatt ID, Pandey MK, Shishodia S, Nair MG: From traditional Ayurvedic medicine to modern medicine: identification of therapeutic targets for suppression of inflammation and cancer. Expert Opin Ther Targets 2006;10:87-118.

105 Guo W, Kong E, Meydani M: Dietary polyphenols, inflammation, and cancer. Nutr Cancer 2009;61:807-810.

106 Nangia-Makker P, Tait L, Shekhar MP, Palomino E, Hogan V, Piechocki MP, Funasaka T, Raz A: Inhibition of breast tumor growth and angiogenesis by a medicinal herb: Ocimum gratissimum. Int J Cancer 2007;121:884-894.

107 Hostanska K, Reichling J, Bommer S, Weber M, Saller R: Hyperforin a constituent of St John's wort (hypericum perforatum l.) extract induces apoptosis by triggering activation of caspases and with hypericin synergistically exerts cytotoxicity towards human malignant cell lines. Eur J Pharm Biopharm 2003;56:121-132.

108 Hostanska K, Jurgenliemk G, Abel G, Nahrstedt A, Saller R: Willow bark extract (BNO1455) and its fractions suppress growth and induce apoptosis in human colon and lung cancer cells. Cancer Detect Prev 2007;31:129-139.

109 Hostanska K, Daum G, Saller R: Cytostatic and apoptosis-inducing activity of boswellic acids toward malignant cell lines in vitro. Anticancer Res 2002;22:2853-2862.

110 Yamada H, Saiko I (eds): Juzen-Taiho-To (ShiQuan-Da-Bu-Tang). Scientific Evaluations and Clinical Applications. Baton Rouge, CRC Press, 2005.

111 Chia JS, Du JL, Hsu WB, Sun A, Chiang CP, Wang WB: Inhibition of metastasis, angiogenesis, and tumor growth by Chinese herbal cocktail Tien-Hsien liquid. BMC Cancer 2010;10:175.

112 Melzer J, Brignoli R, Diehm C, Reichling J, Do $\mathrm{DD}$, Saller R: Treating intermittent claudication with Tibetan medicine PADMA 28: does it work? Atherosclerosis 2006;189:39-46.

113 Samadi S, Khadivzadeh T, Emami A, Moosavi NS, Tafaghodi M, Behnam HR: The effect of Hypericum perforatum on the wound healing and scar of cesarean. J Altern Complement Med 2010;16:113-117.

114 Dell'Aica I, Niero R, Piazza F, Cabrelle A, Sartor L, Colalto C, Brunetta E, Lorusso G, Benelli R, Albini A, Calabrese F, Agostini C, Garbisa S: Hyperforin blocks neutrophil activation of matrix metalloproteinase-9, motility and recruitment, and restrains inflammation-triggered angiogenesis and lung fibrosis. J Pharmacol Exp Ther 2007;321:492-500.
115 Hostanska K, Bommer S, Weber M, Krasniqi B, Saller R: Comparison of the growth-inhibitory effect of Hypericum perforatum l. extracts, differing in the concentration of phloroglucinols and flavonoids, on leukaemia cells. J Pharm Pharmacol 2003;55:973-980.

116 Suntar I, Akkol EK, Keles H, Oktem A, Baser $\mathrm{KH}$, Yesilada E: A novel wound healing ointment: a formulation of Hypericum perforatum oil and sage and oregano essential oils based on traditional Turkish knowledge. J Ethnopharmacol 2011;134:89-96.

117 Suntar IP, Akkol EK, Yilmazer D, Baykal T, Kirmizibekmez H, Alper M, Yesilada E: Investigations on the in vivo wound healing potential of Hypericum perforatum $l$. J Ethnopharmacol 2010;127:468-477.

118 Linde K: St. John's wort - an overview. Forsch Komplementmed 2009;16:146-155.

119 Linde K, Knuppel L: Large-scale observational studies of Hypericum extracts in patients with depressive disorders - a systematic review. Phytomedicine 2005;12:148-157.

120 Rahimi R, Nikfar S, Abdollahi M: Efficacy and tolerability of Hypericum perforatum in major depressive disorder in comparison with selective serotonin reuptake inhibitors: a meta-analysis. Prog Neuropsychopharmacol Biol Psychiatry 2009;33:118-127.

121 Yang CS, Wang X, Lu G, Picinich SC: Cancer prevention by tea: animal studies, molecular mechanisms and human relevance. Nat Rev Cancer 2009;9:429-439.

122 Saller R, Melzer J, Reichling J, Brignoli R, Meier $\mathrm{R}$ : An updated systematic review of the pharmacology of silymarin. Forsch Komplementmed 2007;14:70-80.

123 Sagar SM: Future directions for research on Sily bum marianum for cancer patients. Integr Cancer Ther 2007;6:166-173.

124 Ramasamy K, Agarwal R: Multitargeted therapy of cancer by silymarin. Cancer Lett 2008;269:352362

125 Comelli MC, Mengs U, Schneider C, Prosdocim M: Toward the definition of the mechanism of action of silymarin: activities related to cellular protection from toxic damage induced by chemotherapy. Integr Cancer Ther 2007;6:120-129.

126 Saller R, Brignoli R, Melzer J, Meier R: An updated systematic review with meta-analysis for the clinical evidence of silymarin. Forsch Komplementmed 2008;15:9-20.

127 Tamayo C, Diamond S: Review of clinical trials evaluating safety and efficacy of milk thistle (Silybum marianum [l.] Gaertn.). Integr Cancer Ther 2007;6:146-157.

$128 \mathrm{Ou}$ B, Huang D, Hampsch-Woodill M, Flanagan JA: When east meets west: The relationship between yin-yang and antioxidation-oxidation. FASEB J 2003;17:127-129.

129 Khatami M: 'Yin and yang' in inflammation: duality in innate immune cell function and tumorigenesis. Expert Opin Biol Ther 2008;8:1461-1472. 